\title{
A general fixed point theorem for pairs of weakly compatible mappings in $G$-metric spaces
}

\author{
Valeriu Popa ${ }^{a, *}$, Alina-Mihaela Patriciu ${ }^{b}$ \\ ${ }^{a}$ Department of Mathematics, Informatics and Educational Sciences, Faculty of Sciences "Vasile Alecsandri" University of Bacău, \\ 157 Calea Mărășești, Bacău, 600115, Romania. \\ ${ }^{b}$ Department of Mathematics, Informatics and Educational Sciences, Faculty of Sciences "Vasile Alecsandri" University of Bacău, \\ 157 Calea Mărășești, Bacău, 600115, Romania.
}

This paper is dedicated to Professor Ljubomir Ćirić

Communicated by Professor V. Berinde

\begin{abstract}
In this paper a general fixed point theorem in $G$-metric spaces for weakly compatible mappings is proved, theorem which generalize the results from Abbas et. al. [M. Abbas and B. E. Rhoades, Appl. Math. and Computation 215 (2009), 262 - 269] and [M. Abbas, T. Nazir and S. Radanović, Appl. Math. and Computation 217 (2010), 4094 - 4099]. In the last part of this paper it is proved that the fixed point problem for these mappings is well posed.(C)2012 NGA. All rights reserved.
\end{abstract}

Keywords: $G$-metric space, weakly compatible mappings, fixed point.

2010 MSC: Primary 54H25; Secondary 47H10.

\section{Introduction}

Let $(X, d)$ be a metric space and $S, T:(X, d) \rightarrow(X, d)$ be two mappings. In 1994, Pant 22] introduced the notion of pointwise $\mathrm{R}$ - weakly commuting mappings. It is proved in 23 ] that the notion of pointwise $\mathrm{R}$ - weakly commutativity is equivalent to commutativity in coincidence points. Jungck [11 defined $S$ and $T$ to be weakly compatible if $S x=T x$ implies $S T x=T S x$. Thus, $S$ and $T$ are weakly compatible if and only if $S$ and $T$ are pointwise $\mathrm{R}$ - weakly commuting.

In [9] and [10], Dhage introduced a new class of generalized metric spaces, named D - metric space. Mustafa and Sims [14], 15] proved that most of the claims concerning the fundamental topological structures

\footnotetext{
*Corresponding author

Email addresses: vpopa@ub.ro (Valeriu Popa), alina.patriciu@ub.ro (Alina-Mihaela Patriciu)
} 
on D - metric spaces are incorrect and introduced appropriate notion of generalized metric space, named G - metric space. In fact, Mustafa, Sims and other authors studied many fixed point results for self mappings in $\mathrm{G}$ - metric spaces under certain conditions [6], [16] - 21], 33] and other papers.

In [25] and [26], Popa initiated the study of fixed points for mappings satisfying implicit relations.

The notion of well posedness of a fixed point problem has generated much interest to several mathematicians, for example [8], [12], [24], [29], [30], [31]. Recently, Popa [27], [33] and Akkouchi and Popa [3], 4], [5] studied well posedness problem for mappings satisfying implicit relations in metric spaces.

The purpose of this paper is to prove a general fixed point theorem in G - metric spaces for weakly compatible pairs of mappings satisfying an implicit relation which generalize the results from [1] and [13]. In the last part of this paper we define the notion of a fixed point problem in $G$ - metric spaces for two mappings and we prove that in $\mathrm{G}$ - metric space with a $\mathrm{G}$ - symmetric, the fixed point problem is well posed.

\section{Preliminaries}

Definition 2.1 ([15]). Let $X$ be a nonempty set and $G: X^{3} \rightarrow \mathbb{R}_{+}$be a function satisfying the following properties:

$\left(G_{1}\right): G(x, y, z)=0$ if $x=y=z$,

$\left(G_{2}\right): 0<G(x, x, y)$ for all $x, y \in X$ with $x \neq y$,

$\left(G_{3}\right): G(x, x, y) \leq G(x, y, z)$ for all $x, y, z \in X$ with $z \neq y$,

$\left(G_{4}\right): G(x, y, z)=G(x, z, y)=G(y, z, x)=\ldots$ (symmetry in all three variables),

$\left(G_{5}\right): G(x, y, z) \leq G(x, a, a)+G(a, y, z)$ for all $x, y, z, a \in X$.

Then the function $G$ is called a $G$ - metric on $X$ and the pair $(X, G)$ is called a $G$ - metric space.

Note that $G(x, y, z)=0$, then $x=y=z$.

Definition $2.2([15])$. Let $(X, G)$ be a metric space. A sequence $\left(x_{n}\right)$ in $X$ is said to be

a) $G$ - convergent if for $\varepsilon>0$, there is an $x \in X$ and $k \in \mathbb{N}$ such that for all $m, n \geq k, G\left(x, x_{n}, x_{m}\right)<\varepsilon$.

b) $G$ - Cauchy if for each $\varepsilon>0$, there exists $k \in \mathbb{N}$ such that for all $n, m, p \geq k, G\left(x_{n}, x_{m}, x_{p}\right)<\varepsilon$, that is $G\left(x_{n}, x_{m}, x_{p}\right) \rightarrow 0$ as $m, n, n \rightarrow \infty$.

c) A $G$ - metric space is said to be $G$ - complete if every $G$ - Cauchy sequence is $G$ - convergent.

Lemma $2.3([15])$. Let $(X, G)$ be a $G$ - metric space. Then, the following properties are equivalent:

1) $\left(x_{n}\right)$ is $G$ - convergent to $x$;

2) $G\left(x_{n}, x_{n}, x\right) \rightarrow 0$ as $n \rightarrow \infty$;

3) $G\left(x_{n}, x, x\right) \rightarrow 0$ as $n \rightarrow \infty$;

4) $G\left(x_{m}, x_{n}, x\right) \rightarrow 0$ as $m, n \rightarrow \infty$.

Lemma $2.4([15])$. If $(X, G)$ is a $G$ - metric space, the following are equivalent:

1) $\left(x_{n}\right)$ is $G$ - Cauchy.

2) For every $\varepsilon>0$, there is $k \in \mathbb{N}$ such that $G\left(x_{n}, x_{m}, x_{m}\right)<\varepsilon$ for all $n, m \geq k$.

Definition 2.5 ([14]). Let $(X, G)$ and $\left(X^{\prime}, G^{\prime}\right)$ be two $G$ - metric spaces. A function $f:(X, G) \rightarrow\left(X^{\prime}, G^{\prime}\right)$ is said to be $G$ - continuous at a point $x \in X$ if for $\varepsilon>0$, there exists $\delta>0$ such that for all $x, y \in X$ and $G(a, x, y)<\delta$, then $G^{\prime}(f(a), f(x), f(y))<\varepsilon$.

A function $f$ is $G$ - continuous if $f$ is $G$ - continuous at each $x \in X$.

Lemma $2.6([15])$. Let $(X, G)$ and $\left(X^{\prime}, G^{\prime}\right)$ be $G$ - metric spaces. Then, a function $f:(X, G) \rightarrow\left(X^{\prime}, G^{\prime}\right)$ is $G$ - continuous at a point $x \in X$ if and only if it is $G$ - sequentially continuous, that is, whenever $\left(x_{n}\right)$ is $G$ - convergent to $x$, we have that $f\left(x_{n}\right)$ is $G$ - convergent to $f(x)$.

Lemma $2.7([15])$. Let $(X, G)$ be a $G$ - metric space, then the function $G(x, y, z)$ is jointly continuous in all three of its variables.

Definition 2.8 ([15]). A $G$ - metric space $(X, G)$ is called symmetric if $G(x, y, y)=G(y, x, x$, for all $x, y \in X$.

Remark 2.9. There exists $G$ - metric space which is not symmetric (Example 1 [15]). 


\section{Implicit relations}

Definition 3.1. Let $\mathfrak{F}_{G}$ be the set of all continuous functions $F\left(t_{1}, \ldots, t_{6}\right): \mathbb{R}_{+}^{6} \rightarrow \mathbb{R}$ such that $\left(F_{1}\right): F$ is nonincreasing in variable $t_{5}$,

$\left(F_{2}\right)$ : There exists $h_{1} \in[0,1)$ such that for all $u, v \geq 0, F(u, v, v, u, u+v, 0) \leq 0$ implies $u \leq h_{1} v$.

$\left(F_{3}\right)$ : There exists $h_{2} \in[0,1)$ such that for all $t, t^{\prime}>0, F\left(t, t, 0,0, t, t^{\prime}\right)<0$ implies $t \leq h_{2} t^{\prime}$.

Example 3.2. $F\left(t_{1}, \ldots, t_{6}\right)=t_{1}-a t_{2}-b t_{3}-c t_{4}-d t_{5}-e t_{6}$, where $a, b, c, d, e \geq 0$ and $0<a+b+c+2 d+e<1$.

$\left(F_{1}\right)$ : Obviously.

$\left(F_{2}\right)$ : Let $u, v \geq 0$ be and $F(u, v, v, u, u+v, 0)=u-a v-b v-c u-d(u+v) \leq 0$. Then, $u \leq h_{1} v$, where $0 \leq h_{1}=\frac{a+b+\bar{d}}{1-(c+d)}<1$.

$\left(F_{3}\right):$ Let $t, t^{\prime}>0$ and $F\left(t, t, 0,0, t, t^{\prime}\right)=t-a t-d t-e t^{\prime} \leq 0$. Then $t \leq h_{2} t^{\prime}$, where $0 \leq h_{2}=$ $\frac{e}{1-(a+d)}<1$.

Example 3.3. $F\left(t_{1}, \ldots, t_{6}\right)=t_{1}-k \max \left\{t_{2}, t_{3}, t_{4}, t_{5}, t_{6}\right\}$, where $k \in\left[0, \frac{1}{2}\right)$.

$\left(F_{1}\right)$ : Obviously.

$\left(F_{2}\right):$ Let $u, v \geq 0$ be and $F(u, v, v, u, u+v, 0)=u-k \max \{u, v, u+v\} \leq 0$. Hence, $u \leq h_{1} v$, where $0 \leq h_{1}=\frac{k}{1-k}<1$.

$\left(F_{3}\right)$ : Let $t, t^{\prime}>0$ and $F\left(t, t, 0,0, t, t^{\prime}\right)=t-k \max \left\{t, t^{\prime}\right\} \leq 0$. If $t>t^{\prime}$, then $t(1-k) \leq 0$, a contradiction. Hence, $t \leq t^{\prime}$ which implies $t \leq h_{2} t^{\prime}$, where $0 \leq h_{2}=k<1$.

Example 3.4. $F\left(t_{1}, \ldots, t_{6}\right)=t_{1}-k \max \left\{t_{2}, t_{3}, t_{4}, \frac{t_{5}+t_{6}}{2},\right\}$, where $k \in[0,1)$.

$\left(F_{1}\right)$ : Obviously.

$\left(F_{2}\right):$ Let $u, v \geq 0$ be and $F(u, v, v, u, u+v, 0)=u-k \max \left\{u, v, \frac{u+v}{2}\right\} \leq 0$. If $u>v$, then $u(1-k) \leq 0$, a contradiction. Hence, $u \leq v$ which implies $u \leq h_{1} v$, where $0 \leq h_{1}=k<1$.

$\left(F_{3}\right)$ : Let $t, t^{\prime}>0$ and $F\left(t, t, 0,0, t, t^{\prime}\right)=t-k \max \left\{t, \frac{t+t^{\prime}}{2}\right\} \leq 0$. If $t>t^{\prime}$, then $t(1-k) \leq 0$, a contradiction. Hence, $t \leq t^{\prime}$ which implies $t \leq h_{2} t^{\prime}$, where $0 \leq h_{2}=k<1$.

Example 3.5. $F\left(t_{1}, \ldots, t_{6}\right)=t_{1}^{2}-t_{1}\left(a t_{2}+b t_{3}+c t_{4}\right)-d t_{5} t_{6} \leq 0$, where $a, b, c, d \geq 0$ and $0 \leq a+b+c+d<1$.

$\left(F_{1}\right)$ : Obviously.

$\left(F_{2}\right):$ Let $u, v \geq 0$ be and $F(u, v, v, u, u+v, 0)=u^{2}-u(a v+b v+c u) \leq 0$. If $u>0$, then $u-a v-b v-c u \leq 0$ which implies $u \leq h_{1} v$, where $0 \leq h_{1}=\frac{a+b}{1-c}<1$. If $u=0$ then $u \leq h_{1} v$.

Example 3.6. $F\left(t_{1}, \ldots, t_{6}\right)=t_{1}-k \max \left\{\frac{t_{3}+t_{4}}{2}, \frac{t_{5}+t_{6}}{2}\right\}$, where $k \in[0,1)$.

$\left(F_{1}\right)$ : Obviously.

$\left(F_{2}\right):$ Let $u, v \geq 0$ be such that $F(u, v, v, u, u+v, 0)=u-k \max \left\{v, \frac{u+v}{2}\right\} \leq 0$. If $u>v$, then $u(1-k) \leq 0$, a contradiction. Hence, $u \leq v$ which implies $u \leq h_{1} v$, where $0 \leq h_{1}=k<1$.

$\left(F_{3}\right): F\left(t, t, 0,0, t, t^{\prime}\right)=t-k \max \left\{t, \frac{t+t^{\prime}}{2}\right\} \leq 0$.If $t>t^{\prime}$ then $t(1-k) \leq 0$, a contradiction. Hence $t \leq t^{\prime}$ which implies $t \leq h_{2} t^{\prime}$, where $0 \leq h_{2}=k<1$.

Example 3.7. $F\left(t_{1}, \ldots, t_{6}\right)=t_{1}^{3}-c \frac{t_{3}^{2} t_{4}^{2}+t_{5}^{2} t_{6}^{2}}{1+t_{2}+t_{3}+t_{4}}$, where $c \in[0,1)$.

$\left(F_{1}\right)$ : Obviously. 
$\left(F_{2}\right):$ Let $u, v \geq 0$ be and $F(u, v, v, u, u+v, 0)=u^{3}-c \frac{v^{2} u^{2}}{1+2 v+u} \leq 0$. If $u>0$, then $u \leq c v \frac{v}{1+2 v+u} \leq$ $c v$. Hence, $u \leq h_{1} v$, where $0 \leq h_{1}=c<1$. If $u=0$, then $u \leq h_{1} v$.

$\left(F_{3}\right)$ : Let $t, t^{\prime}>0$ be such that $F\left(t, t, 0,0, t, t^{\prime}\right)=t^{3}-c \frac{t^{2} t^{\prime 2}}{1+t} \leq 0$, which implies $t^{2}-c \frac{t}{1+t} t^{\prime 2} \leq c t^{\prime 2}$. Hence $t \leq h_{2} t^{\prime}$, where $0 \leq h_{2}=\sqrt{c}<1$. If $u=0$ then $u \leq h_{1} v$.

Example 3.8. $F\left(t_{1}, \ldots, t_{6}\right)=t_{1}^{2}-a t_{2}^{2}-b \frac{t_{5} t_{6}}{1+t_{3}^{2}+t_{4}^{2}}$, where $a, b \geq 0$ and $0 \leq a+b<1$.

$\left(F_{1}\right)$ : Obviously.

$\left(F_{2}\right)$ : Let $u, v \geq 0$ be and $F(u, v, v, u, u+v, 0)=u^{2}-a v^{2} \leq 0$. Hence, $u \leq h_{1} v$, where $0 \leq h_{1}=\sqrt{a}<1$.

$\left(F_{3}\right)$ : Let $t, t^{\prime}>0$ be and $F\left(t, t, 0,0, t, t^{\prime}\right)=t^{2}-a t^{2}-b t t^{\prime} \leq 0$, which implies $t \leq h_{2} t^{\prime}$, where $0 \leq h_{2}=$ $\frac{b}{1-a}<1$.

Example 3.9. $F\left(t_{1}, \ldots, t_{6}\right)=t_{1}-a t_{2}-b t_{3}-c \max \left\{2 t_{4}, t_{5}+t_{6}\right\}$, where $a, b, c \geq 0$ and $0 \leq a+b+2 c<1$.

$\left(F_{1}\right)$ : Obviously.

$\left(F_{2}\right)$ : Let $u, v \geq 0$ be and $F(u, v, v, u, u+v, 0)=u-a v-c \max \{2 u, u+v\} \leq 0$. If $u>v$, then $u(1-(a+b+2 c)) \leq 0$, a contradiction. Hence, $u \leq v$ which implies $u \leq h_{1} v$, where $0 \leq h_{1}=\frac{a+b+c}{1-c}<1$.

$\left(F_{3}\right)$ : Let $t, t^{\prime}>0$ be and $F\left(t, t, 0,0, t, t^{\prime}\right)=t-a t-c\left(t+t^{\prime}\right) \leq 0$, which implies $t \leq h_{2} t^{\prime}$, where $0 \leq h_{2}=\frac{c}{1-(a+c)}<1$.

Example 3.10. $F\left(t_{1}, \ldots, t_{6}\right)=t_{1}-a t_{2}-b t_{3}-c \max \left\{t_{4}+t_{5}, 2 t_{6}\right\}$, where $a, b, c \geq 0$ and $0 \leq a+b+3 c<1$.

$\left(F_{1}\right)$ : Obviously.

$\left(F_{2}\right):$ Let $u, v \geq 0$ be and $F(u, v, v, u, u+v, 0)=u-a v-b v-c(2 u+v) \leq 0$, which implies $u \leq h_{1} v$, where $0 \leq h_{1}=\frac{a+b+c}{1-2 c}<1$.

$\left(F_{3}\right)$ : Let $t, t^{\prime}>0$ be and $F\left(t, t, 0,0, t, t^{\prime}\right)=t-a t-c \max \left\{t, 2 t^{\prime}\right\}$. If $t>2 t^{\prime}$ then $t(1-a-c) \leq 0$, a contradiction. Hence $t \leq 2 t^{\prime}$ which implies $t \leq h_{2} t^{\prime}$, where $0 \leq h_{2}=\frac{2 c}{1-a}<1$.

Example 3.11. $F\left(t_{1}, \ldots, t_{6}\right)=t_{1}-c \max \left\{t_{2}, t_{3}, \sqrt{t_{4} t_{6}}, \sqrt{t_{5} t_{6}}\right\}$, where $c \in[0,1)$.

$\left(F_{1}\right)$ : Obviously.

$\left(F_{2}\right)$ : Let $u, v \geq 0$ be such that $F(u, v, v, u, u+v, 0)=u-c v \leq 0$, which implies $u \leq h_{1} v$, where $0 \leq h_{1}=c<1$.

$\left(F_{3}\right)$ : Let $t, t^{\prime}>0$ be and $F\left(t, t, 0,0, t, t^{\prime}\right)=t-c \max \left\{t, \sqrt{t t^{\prime}}\right\} \leq 0$. If $t>t^{\prime}$ then $t(1-c) \leq 0$, a contradiction. Hence $t \leq t^{\prime}$ which implies $t \leq h_{2} t^{\prime}$, where $0 \leq h_{2}=c<1$.

Example 3.12. $F\left(t_{1}, \ldots, t_{6}\right)=t_{1}-k \max \left\{t_{2}, t_{3}, t_{4}, \frac{2 t_{4}+t_{6}}{3}, \frac{2 t_{4}+t_{3}}{3}, \frac{t_{5}+t_{6}}{3}\right\}$, where $k \in[0,1)$.

$\left(F_{1}\right)$ : Obviously.

$\left(F_{2}\right)$ : Let $u, v \geq 0$ be such that

$$
F(u, v, v, u, u+v, 0)=u-k \max \left\{u, v, \frac{2 u}{3}, \frac{2 u+v}{3}, \frac{u+v}{3}\right\} \leq 0 .
$$

If $u>v$, then $u(1-k) \leq 0$, a contradiction. Hence $u \leq v$ which implies $u \leq h_{1} v$, where $0 \leq h_{1}=k<1$. $\left(F_{3}\right):$ Let $t, t^{\prime}>0$ be and $F\left(t, t, 0,0, t, t^{\prime}\right)=t-k \max \left\{t, \frac{t^{\prime}}{3}, \frac{t+t^{\prime}}{3}\right\}$. If $t>t^{\prime}$ then $t(1-k) \leq 0$, a contradiction. Hence $t \leq t^{\prime}$ which implies $t \leq h_{2} t^{\prime}$, where $0 \leq h_{2}=k<1$. 


\section{General fixed point theorem}

Definition 4.1. Let $f$ and $g$ be self maps of a nonempty set $X$. If $w=f x=g x$ for some $x \in X$, then $x$ is called a coincidence point of $f$ and $g$ and $w$ is called a point of coincidence of $f$ and $g$.

Lemma $4.2([1])$. Let $f$ and $g$ be weakly compatible self mappings of nonempty set $X$. If $f$ and $g$ have a unique point of coincidence $w=f x=g x$, then $w$ is the unique common fixed point of $f$ and $g$.

Lemma 4.3. Let $(X, G)$ be a $G$ - metric space and $f, g:(X, G) \rightarrow(X, G)$ two functions such that

$$
\begin{gathered}
F(G(f x, f y, f y), G(g x, g y, g y), G(g x, f x, f x), G(g y, f y, f y), \\
G(g x, f y, f y), G(g y, f x, f x)) \leq 0
\end{gathered}
$$

for all $x, y \in X$ and $F$ satisfying property $\left(F_{3}\right)$. Then, $f$ and $g$ have at most a point of coincidence.

Proof. Suppose that $u=f p=g p$ and $v=f q=g q$. Then by (4.1) we have

$$
\begin{gathered}
F(G(f q, f p, f p), G(g q, g p, g p), G(g q, f q, f q), G(g p, f p, f p), \\
G(g q, f p, f p), G(g p, f q, f q)) \leq 0, \\
F(G(g q, g p, g p), G(g q, g p, g p), 0,0, G(g q, g p, g p), G(g q, g p, g p)) \leq 0
\end{gathered}
$$

which implies by $\left(F_{3}\right)$ that

$$
G(g q, g p, g p) \leq h_{2} G(g p, g q, g q)
$$

Similarly, we obtain that

$$
G(g p, g q, g q) \leq h_{2} G(g q, g p, g p)
$$

which implies that $G(g q, g p, g p)\left(1-h_{2}^{2}\right) \leq 0$. Hence $G(g q, g p, g p)=0$, i.e. $g q=g p$. Therefore $u=f p=$ $g p=g q=f q=v$.

Theorem 4.4. Let $(X, G)$ be a $G$ - metric space and $f, g:(X, G) \rightarrow(X, G)$ satisfying inequality (4.1) for all $x, y \in X$, where $F \in \mathfrak{F}_{G}$. If $f(X) \subset g(X)$ and $g(X)$ is a $G$ - complete metric subspace of $(X, G)$, then $f$ and $g$ have a unique point of coincidence. Moreover, if $f$ and $g$ are weakly compatible, then $f$ and $g$ have a unique common fixed point.

Proof. Let $x_{0}$ be an arbitrary point of $X$ and $x_{1} \in X$ such that $f x_{0}=g x_{1}$. This can be done since $f(X) \subset g(X)$. Continuing this process, having chosen $x_{n}$ in $X$, we obtain $x_{n+1}$ such that $f x_{n}=g x_{n+1}$. Then, by (4.1) we have successively

$$
\begin{gathered}
F\left(G\left(f x_{n-1}, f x_{n}, f x_{n}\right), G\left(g x_{n-1}, g x_{n}, g x_{n}\right), G\left(g x_{n-1}, f x_{n-1}, f x_{n-1}\right),\right. \\
\left.G\left(g x_{n}, f x_{n}, f x_{n}\right), G\left(g x_{n-1}, f x_{n}, f x_{n}\right), G\left(g x_{n}, f x_{n-1}, f x_{n-1}\right)\right) \leq 0, \\
F\left(G\left(g x_{n}, g x_{n+1}, g x_{n+1}\right), G\left(g x_{n-1}, g x_{n}, g x_{n}\right), G\left(g x_{n-1}, g x_{n}, g x_{n}\right),\right. \\
\left.G\left(g x_{n}, g x_{n+1}, g x_{n+1}\right), G\left(g x_{n-1}, g x_{n+1}, g x_{n+1}\right), 0\right) \leq 0 .
\end{gathered}
$$

By $\left(F_{1}\right)$ and $\left(G_{5}\right)$ we obtain

$$
\begin{gathered}
F\left(G\left(g x_{n}, g x_{n+1}, g x_{n+1}\right), G\left(g x_{n-1}, g x_{n}, g x_{n}\right), G\left(g x_{n-1}, g x_{n}, g x_{n}\right),\right. \\
\left.G\left(g x_{n}, g x_{n+1}, g x_{n+1}\right), G\left(g x_{n-1}, g x_{n}, g x_{n}\right)+G\left(g x_{n}, g x_{n+1}, g x_{n+1}\right), 0\right) \leq 0 .
\end{gathered}
$$

By $\left(F_{2}\right)$ we obtain

$$
G\left(g x_{n}, g x_{n+1}, g x_{n+1}\right) \leq h_{1} G\left(g x_{n-1}, g x_{n}, g x_{n}\right)
$$

Continuing the above process we obtain

$$
G\left(g x_{n}, g x_{n+1}, g x_{n+1}\right) \leq h_{1}^{n} G\left(g x_{0}, g x_{1}, g x_{1}\right) .
$$


Then for $m>n$

$$
\begin{aligned}
G\left(g x_{n}, g x_{m}, g x_{m}\right) \leq & G\left(g x_{n}, g x_{n+1}, g x_{n+1}\right)+G\left(g x_{n+1}, g x_{n+2}, g x_{n+2}\right)+ \\
& +\ldots+G\left(g x_{m-1}, g x_{m}, g x_{m}\right) \\
\leq & \left(h_{1}^{n}+h_{1}^{n+1}+\ldots+h_{1}^{m-1}\right) G\left(g x_{0}, g x_{1}, g x_{1}\right) \\
\leq & \frac{h_{1}^{n}}{1-h_{1}} G\left(g x_{0}, g x_{1}, g x_{1}\right)
\end{aligned}
$$

which implies that $G\left(g x_{n}, g x_{m}, g x_{m}\right) \rightarrow 0$ as $n, m \rightarrow \infty$.

Hence, $\left(g x_{n}\right)$ is a $G$ - Cauchy sequence. Since $g(X)$ is $G$ - complete, there exists a point $q$ in $g(X)$ such that $g x_{n} \rightarrow q$ as $n \rightarrow \infty$. Consequently, we can find a point $p \in X$ such that $g p=q$. We prove that $f p=g p$.

By (4.1) we have successively

$$
\begin{gathered}
F\left(G\left(f x_{n-1}, g p, g p\right), G\left(g x_{n-1}, g p, g p\right), G\left(g x_{n-1}, f x_{n-1}, f x_{n-1}\right),\right. \\
\left.G(g p, f p, f p), G\left(g x_{n-1}, f p, f p\right), G\left(g p, f x_{n-1}, f x_{n-1}\right)\right) \leq 0, \\
F\left(G\left(g x_{n}, f p, f p\right), G\left(g x_{n-1}, g p, g p\right), G\left(g x_{n-1}, g x_{n}, g x_{n}\right),\right. \\
\left.G(g p, f p, f p), G\left(g x_{n-1}, f p, f p\right), G\left(g p, g x_{n}, g x_{n}\right)\right) \leq 0 .
\end{gathered}
$$

Letting $n$ tend to infinity, we obtain

$$
F(G(g p, f p, f p), 0,0, G(g p, f p, f p), G(g p, f p, f p), 0) \leq 0 .
$$

By $\left(F_{1}\right)$ it follows that $G(g p, f p, f p)=0$ which implies $g p=f p$. Hence $w=f p=g p$ is a point of coincidence of $f$ and $g$. By Lemma 4.3, $w$ is the unique point of coincidence. Moreover, if $f$ and $g$ are weakly compatible, by Lemma $4.2, w$ is the unique common fixed point of $f$ and $g$.

Remark 4.5. 1) By Example 3.2 with $d=e=0$ and Theorem 4.4 we obtain a partial result from Theorem 2.3 [1].

2) By Example 3.2 for $b=c=d=e=0$ we obtain Theorem 2.1 [13].

3) By Example 3.2 for $b=c=2$ and Theorem 4.4 we obtain a partial result from Theorem 2.6 [1].

4) By Example 3.3 for $h \in\left[0, \frac{1}{2}\right)$ we obtain a partial result of Theorems 2.4, 2.5 [1] which is a form of Ciric result [7] in $G$-metric space.

5) By Examples $3.4-3.12$ we obtain new results.

\section{Well posedness problem of fixed point for two mappings in $G$ - metric spaces}

Definition 5.1. Let $(X, G)$ be a metric space and $f:(X, d) \rightarrow(X, d)$ be a mapping. The fixed point problem $f$ is said to be well posed $[8$ if

1) $f$ has a unique fixed point $x_{0} \in X$,

2) for any sequence $\left(x_{n}\right) \in X$ with $\lim _{n \rightarrow \infty} d\left(x_{n}, f x_{n}\right)=0$ we have

$$
\lim _{n \rightarrow \infty} d\left(x_{n}, x_{0}\right)=0 .
$$

Definition 5.2. A function $F: \mathbb{R}_{+}^{6} \rightarrow \mathbb{R}$ have property $\left(F_{p}\right)$ if for $u, v, w \geq 0$ and $F(u, v, 0, w, u, v) \leq 0$, there exists $p \in(0,1)$ such that $u \leq p \max \{v, w\}$.

Example 5.3. $F\left(t_{1}, \ldots, t_{6}\right)=t_{1}-a t_{2}-b t_{3}-c t_{4}-d t_{5}-e t_{6}$, as in Example 3.2 .

Let $u, v, w \geq 0$ be and $F(u, v, 0, w, u, v)=u-a v-c w-d u-e v \leq 0$ which implies $u \leq p \max \{v, w\}$, where $0<p=\frac{a+c+e}{1-d}<1$. 
Example 5.4. $F\left(t_{1}, \ldots, t_{6}\right)=t_{1}-k \max \left\{t_{2}, \ldots, t_{6}\right\}$, where $k \in\left[0, \frac{1}{2}\right)$.

Let $u, v, w \geq 0$ be and $F(u, v, 0, w, u, v)=u-k \max \{v, w\} \leq 0$. If $u>\max \{v, w\}$, then $u(1-k) \leq 0$, a contradiction. Hence $u \leq \max \{v, w\}$ which implies $u \leq p \max \{v, w\}$, where $0<p=k<1$.

Example 5.5. $F\left(t_{1}, \ldots, t_{6}\right)=t_{1}-k \max \left\{t_{2}, t_{3}, t_{4}, \frac{t_{5}+t_{6}}{2}\right\}$, where $k \in[0,1)$.

Let $u, v, w \geq 0$ be and $F(u, v, 0, w, u, v)=u-k \max \left\{v, w, \frac{1}{2}(u+v)\right\}$. If $u>\max \{v, w\}$, then $u>\frac{u+v}{2}$, which implies $u(1-k) \leq 0$, a contradiction, hence $u \leq \max \{v, w\}$ which implies $u \leq p \max \{v, w\}$, where $0<p=k<1$.

Example 5.6. $F\left(t_{1}, \ldots, t_{6}\right)=t_{1}^{2}-t_{2}\left(a t_{2}+b t_{3}+c t_{4}\right)-d t_{5} t_{6}$, where $a, b, c, d \geq 0$ and $0 \leq a+b+c+d<1$.

Let $u, v, w \geq 0$ be and $F(u, v, 0, w, u, v)=u^{2}-u(a v+c w)-d u v \leq 0$. If $u>0$, then $u \leq p \max \{v, w\}$, where $0 \leq p=a+c+d<1$. If $u=0$, then $u \leq p \max \{v, w\}$.

Example 5.7. $F\left(t_{1}, \ldots, t_{6}\right)=t_{1}-k \max \left\{t_{2}, \frac{t_{3}+t_{4}}{2}, \frac{t_{5}+t_{6}}{2}\right\}$, where $k \in[0,1)$.

Let $u, v, w \geq 0$ be and $F(u, v, 0, w, u, v)=u-k \max \left\{v, \frac{w}{2}, \frac{u+v}{2}\right\}$ which implies $u-k \max \left\{v, \frac{w}{2}, \frac{u+v}{2}\right\} \leq$ 0 . If $u>\max \{v, w\}$, then $u(1-k) \leq 0$, a contradiction. Hence $u \leq \max \{v, w\}$ which implies $u \leq$ $p \max \{v, w\}$, where $0<p=k<1$.

Example 5.8. $F\left(t_{1}, \ldots, t_{6}\right)=t_{1}^{3}-c \frac{t_{3}^{2} t_{4}^{2}+t_{5}^{2} t_{6}^{2}}{1+t_{2}+t_{3}+t_{4}}$, where $c \in[0,1)$.

Let $u, v, w \geq 0$ be and $F(u, v, 0, w, u, v)=u^{3}-c \frac{u^{2} v^{2}}{1+v+w} \leq 0$. If $u>0$, then $u \leq c v \frac{v}{1+v+w} \leq c v \leq$ $p \max \{v, w\}$, where $0<p=c<1$. If $u=0$, then $u \leq p \max \{v, w\}$.

Example 5.9. $F\left(t_{1}, \ldots, t_{6}\right)=t_{1}^{2}-a t_{2}^{2}-c \frac{t_{5} t_{6}}{1+t_{3}^{2}+t_{4}^{2}}$, where $a>0$ and $a+c<1$.

Let $u, v, w \geq 0$ be and $F(u, v, 0, w, u, v)=u^{2}-c \frac{u v}{1+v^{2}} \leq 0$ which implies $u^{2}-a v^{2}-c u v \leq 0$. Let $v>0$, then $f(t)=t^{2}-c t-a$, where $t=\frac{u}{v}$. Then $f(0)<0$ and $f(1)>0$ and hence there exists $p \in(0,1)$ such that $f(t) \leq 0$ for $t \leq p$. Hence $u \leq p v \leq p \max \{v, w\}$. If $v=0$, then $u=0$ and $u \leq p \max \{v, w\}$.

Example 5.10. $F\left(t_{1}, \ldots, t_{6}\right)=t_{1}-a t_{2}-c \max \left\{2 t_{4}, t_{5}+t_{6}\right\}$, where $0 \leq a+2 c<1$.

Let $u, v, w \geq 0$ be and $F(u, v, 0, w, u, v)=u-a v-c \max \{2 w, u+v\}$. If $u>\max \{v, w\}$ then $u(1-a-2 c) \leq 0$, a contradiction. Hence $u \leq \max \{v, w\}$ which implies $u \leq p \max \{v, w\}$, where $0<p=a+2 c<1$.

Example 5.11. $F\left(t_{1}, \ldots, t_{6}\right)=t_{1}-a t_{2}-b t_{3}-c \max \left\{t_{4}+t_{5}, 2 t_{6}\right\} \leq 0$, where $0<p=a+3 c<1$. The proof is similar to the proof of Example 5.8.

Example 5.12. $F\left(t_{1}, \ldots, t_{6}\right)=t_{1}-c \max \left\{t_{2}, t_{3}, \sqrt{t_{4} t_{6}}, \sqrt{t_{5} t_{6}}\right\}$, where $c \in[0,1)$.

Let $u, v, w \geq 0$ be and $F(u, v, 0, w, u, v)=u-c \max \{v, \sqrt{v w}, \sqrt{u v}\} \leq 0$. If $u>\max \{v, w\}$ then $u(1-c) \leq 0$, a contradiction. Hence $u \leq \max \{v, w\}$ which implies $u \leq p \max \{v, w\}$, where $0<p=c<1$.

Example 5.13. $F\left(t_{1}, \ldots, t_{6}\right)=t_{1}-k \max \left\{t_{2}, t_{3}, t_{4}, \frac{2 t_{4}+t_{6}}{3}, \frac{2 t_{4}+t_{5}}{3}, \frac{t_{5}+t_{6}}{3}\right\}$, where $k \in[0,1)$.

Let $u, v, w \geq 0$ be and $F(u, v, 0, w, u, v)=u-k \max \left\{v, w, \frac{2 w+v}{3}, \frac{2 w}{3}, \frac{u+v}{3}\right\} \leq 0$. If $u>\max \{v, w\}$ then $u(1-k) \leq 0$, a contradiction. Hence $u \leq \max \{v, w\}$ which implies $u \leq p \max \{v, w\}$, where 0 $<p=k<1$. 
Definition 5.14. Let $(X, G)$ be a $G$ - metric space and $f, g:(X, G) \rightarrow(X, G)$. The common fixed problem of $f$ and $g$ is said to be well posed if:

1) $\quad f$ and $g$ have a unique common fixed point,

2) for any sequence $\left(x_{n}\right)$ in $X$ with

$$
\lim _{n \rightarrow \infty} G\left(x_{n}, f x_{n}, f x_{n}\right)=0
$$

and

$$
\lim _{n \rightarrow \infty} G\left(x_{n}, g x_{n}, g x_{n}\right)=0
$$

then

$$
\lim _{n \rightarrow \infty} G\left(x, x_{n}, x_{n}\right)=0 .
$$

Theorem 5.15. Let $(X, G)$ be a symmetric $G$ - metric space. For mappings $f, g:(X, G) \rightarrow(X, G)$ satisfying Theorem 4.4 and $F$ having property $\left(F_{p}\right)$, the fixed point problem of $f$ and $g$ is well posed.

Proof. By Theorem $4.4 f$ and $g$ have a unique common fixed point $x$. Let $\left(x_{n}\right)$ be a sequence in $(X, G)$ such that $\lim _{n \rightarrow \infty} G\left(x_{n}, f x_{n}, f x_{n}\right)=0$ and $\lim _{n \rightarrow \infty} G\left(x_{n}, g x_{n}, g x_{n}\right)=0$. By (4.1) we have successively

$$
\begin{gathered}
F\left(G\left(f x, f x_{n}, f x_{n}\right), G\left(g x, g x_{n}, g x_{n}\right), G(g x, f x, f x),\right. \\
\left.G\left(g x_{n}, f x_{n}, f x_{n}\right), G\left(g x, f x_{n}, f x_{n}\right), G\left(g x_{n}, f x, f x\right)\right) \leq 0, \\
F\left(G\left(x, f x_{n}, f x_{n}\right), G\left(x, g x_{n}, g x_{n}\right), 0, G\left(g x_{n}, f x_{n}, f x_{n}\right),\right. \\
\left.G\left(x, f x_{n}, f x_{n}\right), G\left(g x_{n}, x, x\right)\right) \leq 0 .
\end{gathered}
$$

Since $G$ is a symmetric $G$-metric, $G\left(g x_{n}, x, x\right)=G\left(x, g x_{n}, g x_{n}\right)$ and

$$
\begin{gathered}
F\left(G\left(x, f x_{n}, f x_{n}\right), G\left(x, g x_{n}, g x_{n}\right), 0, G\left(g x_{n}, f x_{n}, f x_{n}\right),\right. \\
\left.G\left(x, f x_{n}, f x_{n}\right), G\left(x, g x_{n}, g x_{n}\right)\right) \leq 0 .
\end{gathered}
$$

By $\left(F_{p}\right)$ we have

$$
\begin{aligned}
G\left(x, f x_{n}, f x_{n}\right) & \leq p \max \left\{G\left(x, g x_{n}, g x_{n}\right), G\left(g x_{n}, f x_{n}, f x_{n}\right)\right\} \\
& \leq p\left(G\left(x, g x_{n}, g x_{n}\right)+G\left(g x_{n}, f x_{n}, f x_{n}\right)\right) .
\end{aligned}
$$

Then by $\left(G_{5}\right)$ and the fact that $(X, G)$ is a symmetric $G$ - metric space we have

$$
\begin{aligned}
G\left(x, x_{n}, x_{n}\right) \leq & G\left(x, f x_{n}, f x_{n}\right)+G\left(f x_{n}, x_{n}, x_{n}\right) \\
\leq & p\left(G\left(x, g x_{n}, g x_{n}\right)+G\left(g x_{n}, f x_{n}, f x_{n}\right)\right)+G\left(f x_{n}, x_{n}, x_{n}\right) \\
\leq & p\left(G\left(x, x_{n}, x_{n}\right)+G\left(x_{n}, g x_{n}, g x_{n}\right)+G\left(g x_{n}, x_{n}, x_{n}\right)+\right. \\
& \left.+G\left(x_{n}, f x_{n}, f x_{n}\right)\right)+G\left(f x_{n}, x_{n}, x_{n}\right) \\
= & p\left(G\left(x, x_{n}, x_{n}\right)+2 G\left(x_{n}, g x_{n}, g x_{n}\right)+\right. \\
& \left.+G\left(x_{n}, f x_{n}, f x_{n}\right)\right)+G\left(f x_{n}, x_{n}, x_{n}\right) .
\end{aligned}
$$

Hence $G\left(x, x_{n}, x_{n}\right) \leq \frac{p+1}{1-p} G\left(x_{n}, f x_{n}, f x_{n}\right)+\frac{2 p}{1-p} G\left(x_{n}, g x_{n}, g x_{n}\right)$. Letting $n$ tend to infinity we obtain $\lim _{n \rightarrow \infty} G\left(x, x_{n}, x_{n}\right)=0$. Hence the common fixed point problem of $f$ and $g$ is well posed.

Remark 5.16. By Theorem 4.4 and Examples 5.3 - 5.13 we obtain new results.

\section{Acknowledgements:}

The authors thank the referee for the valuable comments and suggestions. 


\section{References}

[1] M. Abbas and B. E. Rhoades, Common fixed point results for noncommuting mappings without continuity in generalized metric spaces, Appl. Math. and Computation 215 (2009), 262 - 269. 1 . 4.5

[2] M. Abbas, T. Nazir and S. Radanović, Some periodic point results in generalized metric spaces, Appl. Math. and Computation 217 (2010), 4094 - 4099.

[3] M. Akkouchi and V. Popa, Well posedness of common fixed point problem for three mappings under strict contractive conditions, Bull. Math. Inform. Physics, Petroleum - Gas Univ. Ploieşti 61, 2 (2009), 1 - 10. 1

[4] M. Akkouchi and V. Popa, Well posedness of a fixed point problem using G - function, Sc. St. Res. Univ. "Vasile Alecsandri" Bacău. Ser. Math. Inform. 20 (2010), 5 - 12. 1

[5] M. Akkouchi and V. Popa, Well posedness of fixed point problem for mappings satisfying an implicit relation, Demonstratio Math. 43, 4 (2010), 923 - 929. 1

[6] R. Chung, T. Kadian, A. Rosie and B. E. Rhoades, Property $(P)$ in $G$ - metric spaces, Fixed Point Theory and Applications 2010(2010) Article ID 401684, 12 pages. 1

[7] L. B. Ciric, A generalization of Banach contractions, Proc. Amer. Math. 45 (1974), 267 - 273. 4.5

[8] F. S. De Blassi et J. Myjak, Sur la porosite de contractions sans point fixe, Comptes Rend. Acad. Sci. Paris 308 (1989), 51 - 54. 1, 5.1

[9] B. C. Dhage, Generalized metric spaces and mappings with fixed point, Bull. Calcutta Math. Soc. 84(1992), 329 - 336. 1

[10] B. C. Dhage, Generalized metric spaces and topological structures I, Anal. St. Univ. Al. I. Cuza, Iasi Ser. Mat. 46, 1(2000), 3 - 24. 1

[11] G. Jungck, Common fixed points for noncontinuous, nonself maps on nonnumeric spaces, Far East J. Math. Sci. 4(2)(1996), 195-215. 1

[12] B. K. Lahiri and P. Das, Well posedness and porosity of certain classes of operators, Demonstratio Math. 38 (2005), 170 - 176. 1

[13] S. Manro, S. S. Bahtia and S. Kumar, Expansion mappings theorems in G-metric spaces, Intern. J. Contemp. Math. Sci. 5(2010), no. 51, 2529 - 2535. 1. 4.5

[14] Z. Mustafa and B. Sims, Some remarks concerning D - metric spaces, Intern. Conf. Fixed Point. Theory and Applications, Yokohama, 2004, 189 - 198. 1

[15] Z. Mustafa and B. Sims, A new approach to generalized metric spaces, J. Nonlinear Convex Analysis 7(2006), 289 - 297. 1 , 2.9

[16] Z. Mustafa, H. Obiedat and F. Awadeh, Some fixed point theorems for mappings on G - complete metric spaces, Fixed Point Theory and Applications 2008(2008) Article ID 189870, 12 pages. 1

[17] Z. Mustafa, W. Shatanawi and M. Bataineh, Fixed point theorem on uncomplete $G$ - metric spaces, J. Math. Statistics 4(4)(2008), $196-201$.

[18] Z. Mustafa and B. Sims, Fixed point theorems for contractive mappings in complete G - metric spaces, Fixed Point Theory and Applications, 2009(2009) Article ID 917175, 10 pages.

[19] Z. Mustafa, W. S. Shatanawi and M. Bataineh, Existence of fixed point results in G - metric spaces, Intern. J. Math. Math. Sci. 2009(2009) Article ID 283028, 10 pages.

[20] Z. Mustafa and H. Obiedat, A fixed point theorem of Reich in $G$ - metric spaces, Cuba A. Math. J. 12(2010), 83 $-93$.

[21] H. Obiedat and Z. Mustafa, Fixed results on a nonsymmetric G - metric spaces, Jordan. J. Math. Statistics 3(2)(2010), 65 - 79. 1

[22] R. P. Pant, Common fixed point for noncommuting mappings, J. Math. And Appl. 188(1994), 436 - 440.1

[23] R. P. Pant, Common fixed point for four mappings, Bull. Calcutta Math. Soc. 9(1998), 281 - 286. 1

[24] A. Petruşel, I. A. Rus, J. C. Yao, Well-posedness in the generalized sense of the fixed point problems for multivalued operators, Taiwanese J.Math. 11, 3(2007), 903 - 912. 1

[25] V. Popa, Fixed point theorems for implicit contractive mappings, Stud. Cerc. St. Ser. Mat., Univ. Bacău 7(1997), 129 - 133. 1

[26] V. Popa, Some fixed point theorems for compatible mappings satisfying implicit relations, Demonstratio Math. 32, 1(1999), 157 - 163. 1

[27] V. Popa, Well posedness of fixed problem in orbitally complete metric spaces, Stud. Cerc. St. Ser. Math. Univ. Bacău 16 (2006), Suppl., 209 - 214. 1

[28] V. Popa, Well posedness of fixed point problem in compact metric spaces, Bull. Math. Inform. Physics Series, Petroleum - Gas Univ. Ploieşti 60, 1 (2008), 1 - 4.

[29] S. Reich and A. J. Zaslavski, Well posedness of fixed point problems, Far East J. Math. Sci. Special volume, Part. III (2001), 393 - 401. 1

[30] I. A. Rus, Generalized contractions and applications, Cluj University Press, Cluj-Napoca, 2008. 1

[31] I. A. Rus, Picard operators and well-posedness of fixed point problems, Studia Univ. "Babeş- Bolyai", Mathematica 52, 3(2007), 147 - 156. 1

[32] I. A. Rus, A. Petruşel, G. Petruşel, Fixed Point Theory, Cluj University Press, Cluj Napoca, 2008. 
[33] W. Shatanawi, Fixed point theory for contractive mappings satisfying $\Phi$ - maps in $G$ - metric spaces, Fixed Point Theory and Applications, 2010(2010) Article ID 181650, 9 pages. 